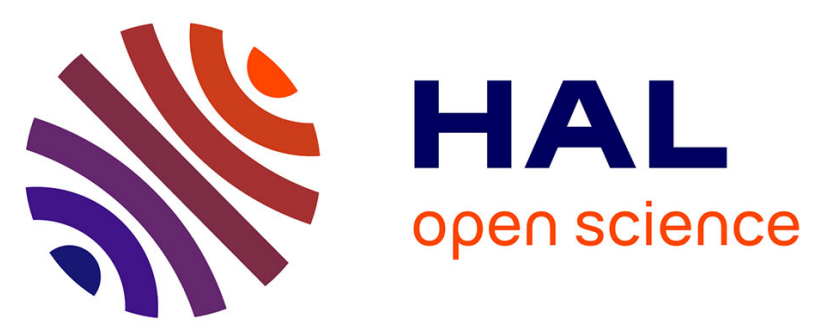

\title{
Nucleation of Magnetization Reversal in Individual Nanosized Nickel Wires
}

W. Wernsdorfer, B. Doudin, D. Mailly, Klaus Hasselbach, A. Benoît, J Meier, J.-Ph Ansermet, B. Barbara

\section{- To cite this version:}

W. Wernsdorfer, B. Doudin, D. Mailly, Klaus Hasselbach, A. Benoît, et al.. Nucleation of Magnetization Reversal in Individual Nanosized Nickel Wires. Physical Review Letters, 1996, 77 (9), pp.1873. hal-01659966

\section{HAL Id: hal-01659966 https://hal.science/hal-01659966}

Submitted on 9 Dec 2017

HAL is a multi-disciplinary open access archive for the deposit and dissemination of scientific research documents, whether they are published or not. The documents may come from teaching and research institutions in France or abroad, or from public or private research centers.
L'archive ouverte pluridisciplinaire HAL, est destinée au dépôt et à la diffusion de documents scientifiques de niveau recherche, publiés ou non, émanant des établissements d'enseignement et de recherche français ou étrangers, des laboratoires publics ou privés. 


\title{
Nucleation of Magnetization Reversal in Individual Nanosized Nickel Wires
}

\author{
W. Wernsdorfer, ${ }^{1,2}$ B. Doudin, ${ }^{3}$ D. Mailly, ${ }^{4}$ K. Hasselbach, ${ }^{1}$ A. Benoit, ${ }^{1}$ J. Meier, ${ }^{3}$ J.-Ph. Ansermet, ${ }^{3}$ and B. Barbara ${ }^{2}$ \\ ${ }^{1}$ Centre de Recherches sur les Très Basses Températures, CNRS, BP166, 38042 Grenoble Cedex 9, France \\ ${ }^{2}$ Laboratoire de Magnétisme Louis Néel, CNRS, BP166, 38042 Grenoble Cedex 9, France \\ ${ }^{3}$ Institut de Physique Expérimentale, Ecole Polytechnique Fédérale de Lausanne, 1015 Lausanne, Switzerland \\ ${ }^{4}$ Laboratoire de Microstructure et Microélectronique, CNRS, 196 Av. H. Ravera, 92220 Bagneux, France
}

(Received 3 January 1996)

The switching of the magnetization of single Ni wires with diameters 40-100 nm was measured at temperatures between 0.13 and $6 \mathrm{~K}$. The angular dependence of the switching field was studied for several wire diameters. Repetitive measurements allow us to obtain histograms of the switching field values. For the smallest diameters, the measurements of the probability of reversal revealed a thermally activated switching following an Arrhenius law with an activation volume much smaller than the volume of the wire. [S0031-9007(96)01046-0]

PACS numbers: 75.60.- d, 75.10.Hk

The mechanisms of magnetization reversal in small magnetic particles have been much discussed in the last decades and prompted intense research activities, motivated in particular by applications in magnetic recording technology [1]. However, experiments were performed, in general, on large assemblies of particles, and the dispersion of morphologies, compositions, orientations, and separations of the magnetic entities limited the interpretation of the results. Furthermore, interactions between particles were difficult to take into account. Single particle studies were possible only in few cases [2]. Recently, insights into the magnetic properties of individual and isolated particles were obtained with the help of near field magnetic force microscopy [3], electron Lorentz microscopy or holography [4], and micro-SQUID (superconducting quantum interference device) magnetometry [5]. It is now possible to make a clear link between experiments performed on nanometer-sized single objects (particles, wires, etc.) and the numerical calculations based on the Brown micromagnetic equations [6].

We report the first study of isolated nanoscale wires with diameters smaller than $100 \mathrm{~nm}$, for which singledomain states could be expected. The cylindrical geometry, with its large shape anisotropy, is well suited for comparison with theory. We obtained unique insight into the process of magnetization reversal by measuring histograms of the switching field as a function of the orientation of the wires in the applied field, their diameter, and the temperature. Furthermore, we measured the probability of switching as a function of the applied field and the temperature. Our studies reveal that the magnetization reversal proceeds by a distortion of the magnetization followed by a nucleation and a propagation process. The observed behavior illustrates the fundamental importance of the study of single, isolated magnetic particles in comparing models and experiments.

We developed planar microbridge dc SQUID [7], made of $\mathrm{Nb}$ (thickness $20 \mathrm{~nm}$ ), which were shown to be able to detect $10^{4} \mu_{B}$ [8]. The SQUID is made of a thin $(20 \mathrm{~nm}) \mathrm{Nb}$ layer in order to prevent flux trapping. The experimental setup allows measurements of hysteresis loops in magnetic fields of up to $0.5 \mathrm{~T}$ and temperatures below $6 \mathrm{~K}$, with a time resolution of $100 \mu \mathrm{s}$.

$\mathrm{Ni}$ wires were produced by electrochemically filling the pores of commercially available nanoporous tracketched polycarbonate membranes of thicknesses of 6 to $10 \mu \mathrm{m}$ [9]. The pore size was chosen in the range of 30 to $100 \mathrm{~nm}$ [10]. In order to place one wire on the SQUID detector, we dissolved the membrane in chloroform and put a drop on a chip of some hundreds of SQUID's. Magnetization measurements were performed on SQUID's with a single isolated wire. Scanning electron microscopy (SEM) (Fig. 1) was used to determine the position and morphology of the wire. The surface roughness was around $5 \mathrm{~nm}$, corresponding to our SEM resolution.

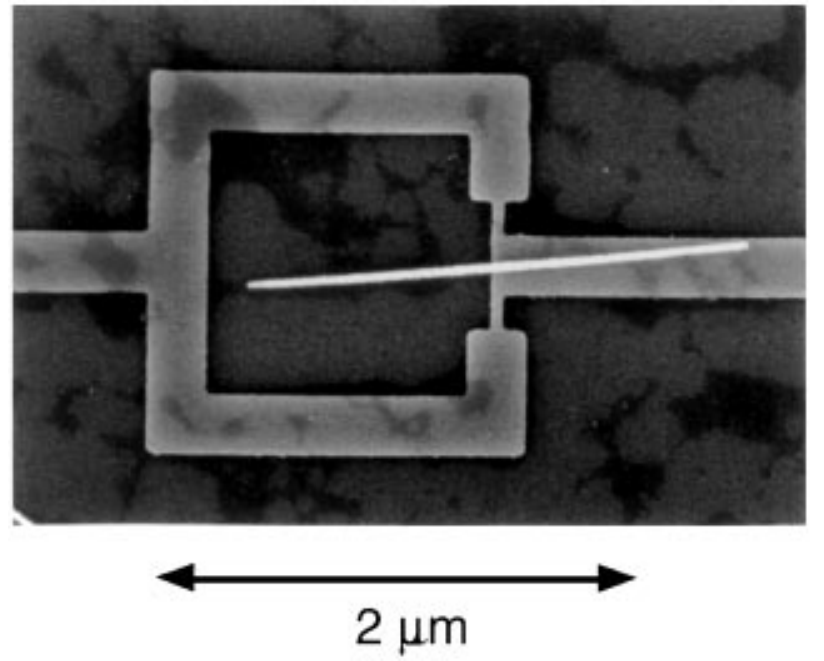

FIG. 1. Scanning electron micrograph (JEOL 6300) of a microbridge dc SQUID and a $\mathrm{Ni}$ wire of diameter of $65 \pm 4 \mathrm{~nm}$. 
The magnetic field was applied in the plane of the SQUID at an angle $\theta$ with respect to the wire axis (easy anisotropy direction), and the flux induced by the wire was detected by the SQUID. If the sample has one end in the SQUID loop (Fig. 1), the signal is approximately proportional to the projection of the magnetization in the direction of the wire axis. This is the reason for the negative slope in the curves in Fig. 2. Under a slowly varying applied field (typically a few $\mathrm{mT} / \mathrm{s}$ ), we observed an abrupt change of the signal, in less than $100 \mu \mathrm{s}$, at the switching field value (Fig. 2).

The measured switching field had the character of a stochastic variable, as expected for sufficiently small wire diameters. We measured histograms of the switching field of several individual wires with diameters between 40 and $100 \mathrm{~nm}$. The angular dependence of the mean switching field $H_{\mathrm{SW}}$ and its standard deviation $\sigma$ of a wire $92 \pm 4 \mathrm{~nm}$ in diameter is shown in Fig. 3. We checked by transmission electron microscopy techniques that our $\mathrm{Ni}$ wires were polycrystalline (typical crystallite size of $10 \mathrm{~nm}$ ), allowing us to suppose that the magnetocrystalline anisotropy was negligible. As the wires have a very high aspect ratio (100:1), we can compare our observations to the predictions of the curling mode of magnetization reversal in an infinite cylinder [11]. In this case, the angular variation of the switching field can be expressed by

$$
H_{\mathrm{SW}}=\frac{M_{S}}{2} \frac{a(1+a)}{\sqrt{a^{2}+(1+2 a) \cos ^{2} \theta}},
$$

where $a=-1.08\left(d_{0} / d\right)^{2}$. The exchange length $d_{0}=$ $2 \sqrt{A} / M_{s}$ ( $A$ is the exchange constant) defines the transition from uniform rotation to curling. By fitting measurements on several wires of diameters between 75 and $100 \mathrm{~nm}$, we found $d_{0}(\mathrm{Ni})=34 \pm 4 \mathrm{~nm}$. This result can be compared favorably to the values commonly cited in

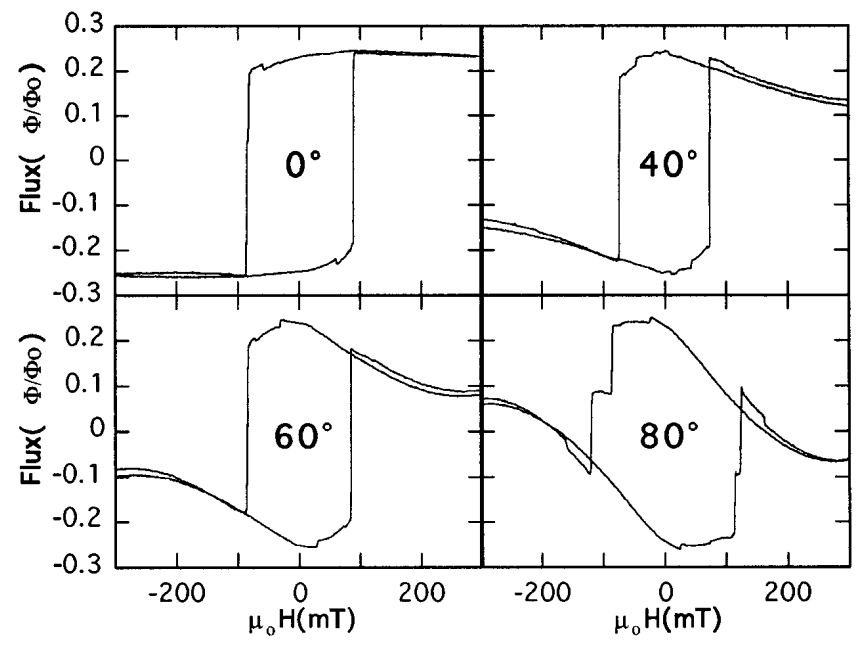

FIG. 2. Typical hysteresis loops of the wire of Fig. 1 (diameter $65 \pm 4 \mathrm{~nm}$ ) at several values of the angle between the applied field and the wire axis. the literature: $d_{0}(\mathrm{Ni})=41 \mathrm{~nm}[6]$. The angular dependence of the switching field of $\mathrm{Ni}$ wires with larger diameters $(100-450 \mathrm{~nm})$ was measured at room temperature by Lederman et al. [12]. The angular dependence they observed was also fitted by Eq. (1), although the deduced value of $d_{0}$ was not as close to the theoretical value.

In repeating hysteresis measurements at a given angle, we obtained the distribution of the switching field values (Fig. 3). It revealed that the switching field histogram can split in some few and distinct peaks, each one corresponding to a different spin configuration with a different energy barrier of nucleation. The width of the histograms varied strongly with the angle $\theta$, but remained always smaller than a few percent of $H_{\mathrm{SW}}$ (Fig. 3).

Measurements performed on wires of diameters smaller than $75 \mathrm{~nm}$ presented an angular variation of the switching field with a new local maximum appearing at $\theta=0$. It can be thought of as the reminiscence of the maximum predicted by the Stoner-Wohlfarth model of uniform rotation [13]. When the sample diameter approaches $d_{0}$, the curling mode is present at small $\theta$ angles, and the uniform rotation occurs at larger $\theta$ values [11]. Our observations (Fig. 4) agree qualitatively with this picture. An alternative theoretical explanation is a reversal of the magnetization by uniform rotation, but affected by the presence of defects of the samples, limiting the height of the maximum at zero angle [14]. This is also qualitative, as our measured values are systematically smaller than the predictions of the two models. The histograms of the switching fields were narrow $\left(0.1 \%\right.$ to $0.4 \%$ of $\left.H_{\mathrm{SW}}\right)$ with a single maximum (Fig. 4).

Extensions of analytical [11] to numerical $[6,15]$ calculations of the micromagnetic equations allow a description of the magnetization reversal process beyond small angle deviations of the magnetization. In cylinders of finite length, the curling mode is immediately followed by the formation of a vortex at one end of the cylinder

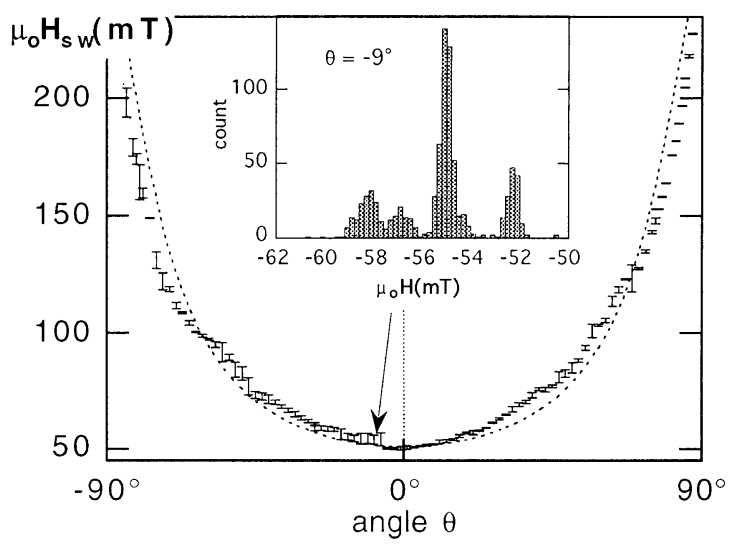

FIG. 3. Angular variation of the switching field of a wire of $\mathrm{Ni}, 92 \pm 4 \mathrm{~nm}$ in diameter, $5 \mu \mathrm{m}$ in length. Bars: width of the histograms. Line: prediction of the curling model. Inset: histogram of the switching field at the angle $\theta=-9^{\circ}$. 


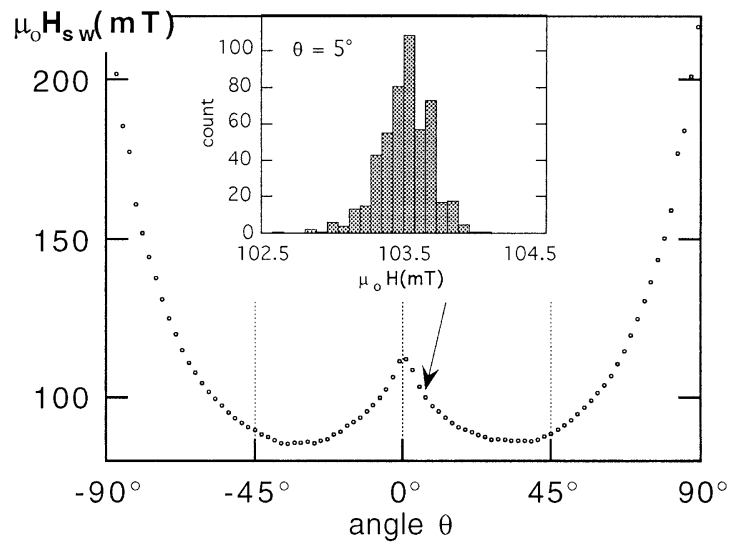

FIG. 4. Angular variation of the switching field of a wire of $\mathrm{Ni}, 50 \pm 5 \mathrm{~nm}$ in diameter, $3.5 \mu \mathrm{m}$ in length. The width of the switching field distribution is smaller than the dot size. Inset: histogram of the switching field at the angle $\theta=5^{\circ}$.

which sweeps across the sample [16]. The concept of the magnetization reversal triggered by a nucleation process has also been recently treated analytically [17]. The complex histograms of the wires of larger diameters indicate that several sites compete for the nucleation, nonetheless with switching fields approaching the value given by the curling model. The narrow histograms of the wires of smaller diameters suggests that a single energy barrier is dominant.

In order to estimate the volume of activation, we performed switching time measurements. At a given temperature, the magnetic field was increased to a set value $H_{W}$, at which we measured the elapsed time until the magnetization switches. This process was repeated about two hundred times, in order to obtain a switching time histogram. The integral of this histogram gave us the switching probability. We fitted the results by a stretched exponential:

$$
P(t)=e^{-(t / \tau)^{\beta}},
$$

where $\tau$ defines the mean waiting time. The case of hopping over a single energy barrier corresponds to $\beta=$ 1. Values of $\beta<1$ correspond to a distribution of energy barriers. We found a value of $\beta$ between 0.1 and 0.5 with wires of diameters between 75 and $100 \mathrm{~nm}$. The wires of smaller diameters had $\beta$ values close to unity (Fig. 5).

We verified that the field and temperature dependence of $\tau$ followed an Arrhenius law $\tau\left(T, H_{W}\right)=$ $\tau_{0} \exp \left[E\left(H_{W}\right) / k T\right]$ with $E\left(H_{W}\right)=E_{0}\left(1-H_{W} / H_{W}^{0}\right) \alpha$ and with $\alpha \approx 1.5$ [18]. We present our data of $\tau\left(T, H_{W}\right)$ in the form of a scaling plot of the applied field values $H_{W}$ as a function of $\left[T \ln \left(\tau / \tau_{0}\right)\right]^{2 / 3}$ (Fig. 6). We found that the data of $\tau\left(T, H_{W}\right)$ obtained at temperatures higher than $1 \mathrm{~K}$ fell on a line provided $\tau_{0} \approx 10^{-8} \mathrm{~s}$ (Fig. 6). Possible explanations for the deviation at temperatures smaller than $1 \mathrm{~K}$ will be discussed elsewhere [19]. The slope and intercept of the scaling plot (Fig. 6) gave

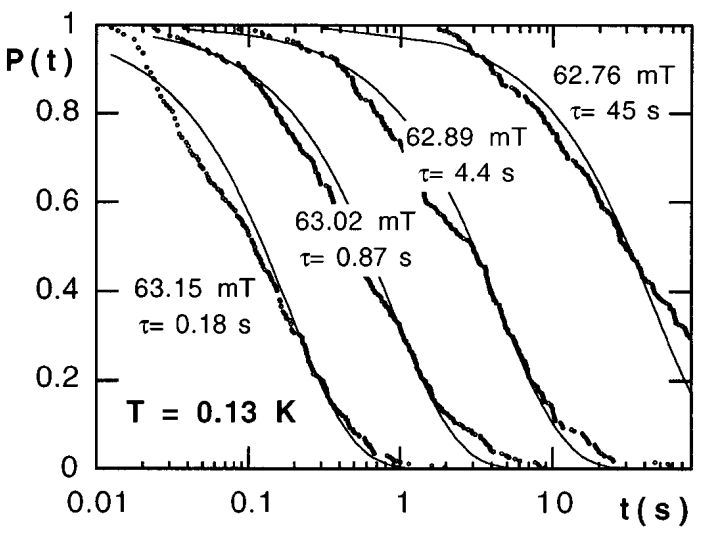

FIG. 5. Probability of not switching of the magnetization as a function of the time at four different applied fields, measured at $0.13 \mathrm{~K}$ for a wire of $\mathrm{Ni}, 45 \pm 5 \mathrm{~nm}$ in diameter at $\theta \approx 30^{\circ}$. Lines: formula (2) with $\beta=1$ and $\tau$ as indicated.

$E_{0} \approx 15000 \mathrm{~K}$ and $H_{W}^{0} \approx 63.5 \mathrm{mT}$. The energy barrier $E_{0}$ can be approximately converted to a thermally activated volume by using $V \approx E_{0} / \mu_{0} M_{S} H_{\mathrm{SW}}=(20 \mathrm{~nm})^{3}$, which is more than 200 times smaller than the wire volume. Therefore, we propose that the magnetization jumps are triggered by a nucleation process. This hypothesis is confirmed by the measurements of the switching field as a function of the external field sweeping rate and of the temperature $[5,18]$.

In conclusion, our measurements on single wires of diameters smaller than $100 \mathrm{~nm}$ give several experimental pieces of evidence that the magnetization reversal in a ferromagnetic wire results from a nucleation and propagation process. For wires with diameters larger than 2 times the exchange length $d_{0}$, we observed that the nucleation occurs at several nearly degenerate fields at values close to the curling instability. For wires of diameters approaching the exchange length, the Stoner-Wohlfarth

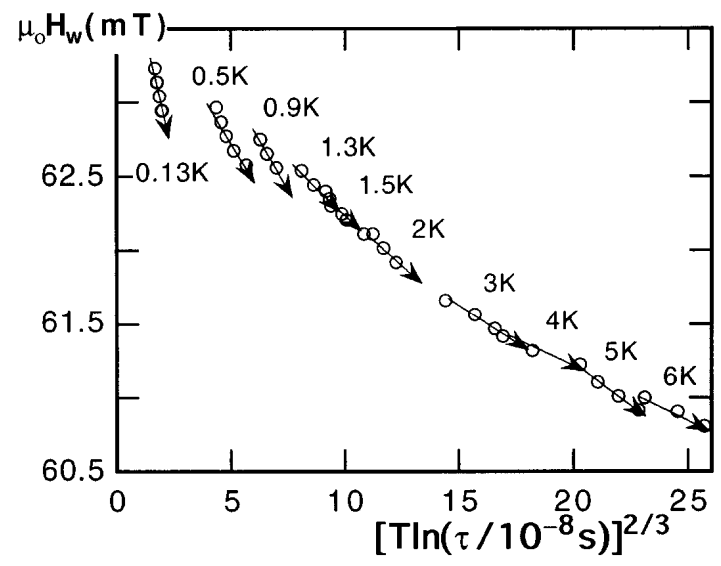

FIG. 6. Scaling plot of the mean switching time $t\left(H_{W}, T\right)$ for several waiting fields $H_{W}$ and temperatures $\left[0.1<t\left(H_{W}, T\right)<\right.$ $60 \mathrm{~s}$. Each arrow is a guide to the eye for data obtained at one temperature and several waiting fields. The field is applied at an angle of $30^{\circ}$ with respect to the wire axes. 
model becomes relevant. In this case, the switching time and switching field measurements reveal that only a single energy barrier is dominant and the reversal process could be described by an Arrhenius law.

We acknowledge the contributions of B. Pannetier and T. Crozes (CRTBT-CNRS) to the success of this work, the hospitality of the Center of Electron Microscopy of the EPFL (CIME), and the help of B. Senior for the electron microscopy pictures. This research was partly financed by the Swiss National Fund, Grant No. 20-42034.94.

[1] H. N. Bertram and J.-G. Zhu, Solid State Physics, edited by H. Ehrenreich and D. Trunbull (Academic, New York, 1992), Vol. 46, p. 271.

[2] J. F. Smyth et al., J. Appl. Phys. 69, 5262 (1991).

[3] M. Lederman, G. A. Gibson, and S. Schultz, J. Appl. Phys. 73, 6961 (1993); M. Lederman, S. Schultz, and M. Ozaki, Phys. Rev. Lett. 73, 1986 (1994); T. Chang and J. G. Chu, J. Appl. Phys. 75, 5553 (1994); M. S. Wei and S. Y. Chou, J. Appl. Phys. 76, 6679 (1994).

[4] T. Hirayama, Q. Ru, T. Tanji, and A. Tonomure, Appl. Phys. Lett. 63, 418 (1993); C. Salling et al., J. Appl. Phys. 75, 7989 (1994); C. Beeli, B. Doudin, and P. Stadelmann, Phys. Rev. Lett. 75, 4630 (1995).

[5] W. Wernsdorfer, K. Hasselbach, D. Mailly, B. Barbara, A. Benoit, L. Thomas, and G. Suran, J. Magn. Magn. Mater. 145, 33 (1995); 151, 38 (1995); Phys. Rev. B 53, 3341 (1996).

[6] M.E. Schabes, J. Magn. Magn. Mater. 95, 249 (1991).

[7] C. Chapelier, M. EL Khatib, P. Perrier, A. Benoit, and D. Mailly, in SQUID 91, Superconducting Devices and
Their Applications, edited by H. Koch and H. Lubbig (Springer-Verlag, Berlin, 1991), pp. 286-291.

[8] W. Wernsdorfer, K. Hasselbach, A. Benoit, B. Barbara, D. Mailly, J. Tuaillon, J. P. Perez, V. Dupuis, J. P. Dupin, G. Guiraud, and A. Perez, J. Appl. Phys. 78, 7192 (1995).

[9] Poretics Corp., 111 Lindbergh Av., Livermore, CA 945509520; Costar Corp. (Nuclepore), One Alewife Center, Cambridge, MA 02140.

[10] B. Doudin and J.-Ph. Ansermet, NanoStructured Mater. 6, 521 (1995).

[11] W.F. Brown, Phys. Rev. 105, 1479 (1957); H. Frei, S. Shtrikman, and D. Treves, Phys. Rev. 106, 446 (1957); S. Shtrikman and D. Treves, J. Phys. Radium 20, 286 (1959); A. Aharoni and S. Shtrikman, Phys. Rev. 10, 1522 (1958); Review: A. Aharoni, Phys. Status Solidi 16, 3 (1966).

[12] M. Lederman, R. O'Barr, and S. Schultz, IEEE Trans. Mag. 31, 3793 (1995); R. O’Barr, M. Lederman, S. Schultz, Weihua $\mathrm{Xu}, \mathrm{A}$. Scherer, and J. Tonucci, J. Appl. Phys. 79, 5303 (1996).

[13] E. C. Stoner and E. P. Wohlfarth, Philos. Trans. London Ser. A 240, 599 (1948); L. Néel, Acad. Sci. Paris 224, 1550 (1947).

[14] H. J. Richter, J. Appl. Phys. 65, 3597 (1989).

[15] W. Chen, D. R. Fredkin, and T. R. Koehler, IEEE 29, 2124 (1993).

[16] M.E. Schabes and H. N. Bertram, J. Appl. Phys. 64, 5832 (1988); B. Yang and D. R. Fredkin, J. Appl. Phys. 79, 5755 (1996).

[17] J. S. Broz, H. B. Braun, O. Brodbeck, W. Baltensperger, and J. S. Helman, Phys. Rev. Lett. 65, 787 (1990); H. B. Braun, ibid., 71, 3557 (1993).

[18] R. H. Victora, Phys. Rev. Lett. 63, 457 (1989).

[19] W. Wernsdorfer et al. (to be published). 